\title{
The impact of urban community hospital surveillance for gonorrhoea on the infection rate and complications in the female A progress report
}

\author{
R. C. RENDTORFF, H. PACKER, S. GLASSCO, AND J. LEVY \\ From the Departments of Community Medicine and Microbiology, University of Tennessee Center for the \\ Health Sciences, and Memphis-Shelby County Health Department, USA
}

SUMMARY This study substantiates a previous report concerning the importance of the urban community hospital, particularly its emergency room, in the detection of gonorrhoea in women. Pelvic inflammatory disease (PID), a major complication of gonorrhoea in women, significantly declined during a nine-year surveillance and control programme in the Memphis-Shelby County area. This suggests that the programme may have prevented PID developing in women through the early detection and treatment of asymptomatic infections.

\section{Introduction}

A report on the community surveillance programme aimed at detecting gonorrhoea in women in Memphis-Shelby County, Tennessee, emphasised the role of the urban community hospital (Rendtorff et al., 1976). The report covered the years 1973-74 and showed that the City of Memphis Hospital (CMH) contributed $53.3 \%$ of all positive cultures of gonorrhoea in only $34.2 \%$ of all tests done in the community, and that the emergency room was an especially productive area. Data for the period 1975-76 are now presented and these are compared with the data for 1973-74.

As our surveillance programme for gonorrhoea in women has now been in existence for a longer period of time than was the case when the previous report was made, an attempt will be made to determine whether the programme has had any impact upon either the rate of gonorrhoea in women participating in our programme, or upon the number of hospital admissions for the most serious complication of gonococcal infection in women-namely, pelvic inflammatory disease (PID).

It is important to demonstrate that the time and effort being devoted to gonorrhoea in present-day venereal disease control programmes are justified by the results. The most valid indices of success

Address for reprints: Dr Rendtorff, 800 Madison Avenue, Box GA150, Memphis, Tennessee 38163, USA

Received for publication 17 August 1977 would be either a stabilisation or a reduction in the infection rate, or statistical evidence that the natura course of gonorrhoeal infections towards PID was being intercepted. The high cost of medical care for women with PID has been studied (Rendtorff et al.; 1974). We feel that careful evaluation should be made of any method which could make an impact on this problem and which might establish guidelines for future control programmes.

\section{Materials and methods}

The methods used in this study have already been described. They consisted of cervical swabs cultured on Thayer-Martin medium incubated in a candle jar at $36^{\circ} \mathrm{C}$, with the exception of specimens from maternity admissions at the $\mathrm{CMH}$ which were vaginal swabs.

One change that might be important is that in 1973-74 specimens from the CMH (and those from emergency room and maternity admissions) were examined in our research project laboratory while other specimens were examined in the Health Department laboratory. During the 1975-76 period, all tests were done in the Health Department laboratory.

Results

Table 1 compares, for each two-year period, the number and percentage of positive cultures according to the place of test. Table 2 compares the 
Table 1 A comparison of results of culture tests for gonorrhoea in women in Memphis-Shelby County for the two-year periods 1973-74 and 1975-76

\begin{tabular}{|c|c|c|c|c|c|c|}
\hline \multirow[b]{2}{*}{ Place of test } & \multicolumn{2}{|c|}{ No. tested } & \multicolumn{2}{|c|}{ No. with positive results } & \multicolumn{2}{|c|}{$\%$ with positive results } \\
\hline & $1973-74$ & $1975-76$ & $1973-74$ & $1975-76$ & $1973-74$ & $1975-76$ \\
\hline \multicolumn{7}{|l|}{ City of Memphis Hospital } \\
\hline Emergency room & 6934 & 7774 & 1685 & 1281 & $24 \cdot 3$ & $16 \cdot 5$ \\
\hline Maternity admissions & 9714 & 12319 & 337 & 370 & $3 \cdot 5$ & $3 \cdot 0$ \\
\hline Obstetrics/gynaecology & 22004 & 24180 & 1075 & 1217 & $4 \cdot 9$ & $5 \cdot 0$ \\
\hline & 38652 & 44273 & 3097 & 2868 & $8 \cdot 0$ & $6 \cdot 5$ \\
\hline \multicolumn{7}{|l|}{ Other community health services } \\
\hline Private hospital emergency room/outpatients & 5400 & 3256 & 108 & 203 & $2 \cdot 0$ & $6 \cdot 2$ \\
\hline Public hospital (inpatients) & 255 & 181 & - & 1 & - & 0.5 \\
\hline Community neighbourhood health centres & 526 & 5515 & 32 & 301 & $6 \cdot 1$ & $5 \cdot 6$ \\
\hline Private physicians & 20233 & 26326 & 332 & 801 & $1 \cdot 6$ & $3 \cdot 0$ \\
\hline Private family planning groups & 3101 & 10533 & 41 & 326 & $1 \cdot 3$ & $3 \cdot 1$ \\
\hline Military dependants & 8009 & 6028 & 58 & 56 & 0.7 & 0.9 \\
\hline Health department family planning clinics & 32726 & 41654 & 2129 & 2350 & $5 \cdot 8$ & $5 \cdot 6$ \\
\hline All others & 161 & 2435 & 9 & 147 & $5 \cdot 6$ & $6 \cdot 0$ \\
\hline & 74411 & 95928 & 2709 & 4185 & $3 \cdot 6$ & $4 \cdot 4$ \\
\hline Total & 113063 & 140201 & 5806 & 7053 & $5 \cdot 1$ & $5 \cdot 0$ \\
\hline
\end{tabular}

Table 2 The percentage of total tests performed in Memphis-Shelby County and the contribution to the total positive cultures, by each place of test for the two-year periods 1973-74 and 1975-76

\begin{tabular}{|c|c|c|c|c|}
\hline \multirow[b]{2}{*}{ Place of test } & \multicolumn{2}{|c|}{ Percentage of total tested } & \multicolumn{2}{|c|}{ Percentage of positive results } \\
\hline & 1973-74 & $1975-76$ & 1973-74 & $1975-76$ \\
\hline \multicolumn{5}{|l|}{ City of Memphis Hospital } \\
\hline Emergency room & $6 \cdot 1$ & $5 \cdot 5$ & $29 \cdot 0$ & $18 \cdot 2$ \\
\hline Maternity admissions & $8 \cdot 6$ & $8 \cdot 8$ & $5 \cdot 8$ & $5 \cdot 2$ \\
\hline Obstetrics/gynaecology & $19 \cdot 5$ & $17 \cdot 2$ & $18 \cdot 5$ & $17 \cdot 3$ \\
\hline & $34 \cdot 2$ & $31 \cdot 5$ & $53 \cdot 3$ & $40 \cdot 6$ \\
\hline \multicolumn{5}{|l|}{ Other community health services } \\
\hline Private hospital emergency room/outpatients & $4 \cdot 8$ & $2 \cdot 3$ & 1.9 & $2 \cdot 9$ \\
\hline Public hospital (inpatients) & $0 \cdot 2$ & $0 \cdot 1$ & - & - \\
\hline Community neighbourhood health centres & $0 \cdot 5$ & 3.9 & $0 \cdot 5$ & $4 \cdot 3$ \\
\hline Private physicians & $17 \cdot 9$ & $18 \cdot 8$ & $5 \cdot 7$ & $11 \cdot 4$ \\
\hline Private family planning groups & $2 \cdot 7$ & $7 \cdot 5$ & 0.7 & $4 \cdot 6$ \\
\hline Military dependants & $7 \cdot 1$ & $4 \cdot 3$ & $1 \cdot 0$ & 0.8 \\
\hline Health department family planning clinics & $32 \cdot 5$ & $29 \cdot 7$ & $36 \cdot 7$ & $33 \cdot 3$ \\
\hline \multirow[t]{2}{*}{ All others } & $0 \cdot 1$ & $P \cdot 1 \cdot 7$ & 0.2 & $2 \cdot 1$ \\
\hline & $65 \cdot 8$ & $68 \cdot 3$ & $46 \cdot 7$ & 59.4 \\
\hline Total & $100 \cdot 0$ & 99.8 & $100 \cdot 0$ & $100 \cdot 0$ \\
\hline
\end{tabular}

percentage of all the tests done with the number of positive cultures for each place of test, thus giving a comparison of the yield from each place in relation to the overall efforts of the surveillance programme.

The total number of tests performed in the community increased from 113063 during the first two-year period (1973-74) to 140201 during the second two-year period (1975-76). However, the percentage of women with positive cultures remained essentially the same during both periods5.1 and $5.0 \%$ respectively (Table 1 ).

There was about a $15 \%$ increase in the number of cultures done at the CMH-from 38652 to 44273 cultures. However, there were fewer positive results at the $\mathrm{CMH}$-from 8.0 to $6.5 \%$. This decline was almost entirely because of a decrease in the rate of positive cultures from the emergency room- from $24 \cdot 3$ to $16 \cdot 5 \%(32 \cdot 1 \%$ decline $)$ in spite of an increase in the number of tests-from 6934 to 7774.

This may reflect a decline in the number of patients with PID seeking care in the emergency room, since patients with symptomatic infections of this type are more likely to seek care there than in any other part of the CMH.

If the total number of positive cultures during the successive two-year periods is considered, the number from the $\mathrm{CMH}$ declined from 3097 to $2868(7 \cdot 4 \%)$. This decline is accounted for entirely by a reduction in the number of positive cultures in the emergency room. On the other hand, the number of positive cultures obtained from other community health services increased from 2709 to $4185(54 \cdot 5 \%)$. The yield of positive cultures from these other c 
services, therefore, markedly increased during the latter period, while the yield from the $\mathrm{CMH}$ declined slightly. Marked increases in the number of positive cultures were noted during the second period from community neighbourhood health centres, private physicians, and private family planning groups; these yielded 1023 more cases and accounted mainly for the increased number of positive cultures from other community health services. This increase was probably due to the fact that new family planning clinics had been established within poor areas, and more private physicians with large practices in these areas joined the surveillance programme. The Health Department also opened new neighbourhood health centers and increased its medical care facilities in these areas of the city. Thus, case-finding efforts were implemented by the other community health services (increase of $35 \%$ ) and $\mathrm{CMH}$ (increase of $15 \%$ ) during the second period. From these combined sources, the percentage of positive cultures for both periods remained approximately the same $(5 \cdot 1$ and $5.0 \%)$.

Table 2 substantiates a previous statement regarding the importance of taking routine cultures for gonorrhoea in women presenting for prenatal care, delivery, or general pelvic complaints in an urban community hospital (Rendtorff et al., 1976). The emergency room showed a decline in the percentage of positive cultures found in the second year, but still continued to provide the highest percentage of positive cultures $(16.5 \%$, Table 1$)$. In terms of percentage of total tests done, the emergency room produced the highest ratio of positive cultures to the number of tests. Thus $18.2 \%$ of total positive cultures were detected in only $5.5 \%$ of tests performed (Table 2 ). $\mathrm{CMH}$ as a whole provided $40.6 \%$ of the positive cultures in only $31 \cdot 5 \%$ of tests.

\section{Discussion}

The second two-year community surveillance data show how important is the urban community hospital in surveillance programmes of gonorrhoea in women, particularly the emergency room as a case-finding source. The significant decline of gonorrhoea in women in the emergency room in spite of intensified testing deserves scrutiny. We do not feel that differences in laboratory practice between the research laboratory responsible for the culturing during the first two-year period and the Health Department Laboratory responsible for the culturing during the second period accounted for this. When the two laboratories were operating simultaneously, comparisons showed their efficacies were about equal.
It is possible that the change reflects a real decline in patients with PID seeking the emergency room during the latter period. This would be an appealing hypothesis to accept as it would indicate some degree of success towards the main objective of the programme-namely, to reduce the female reservoir of infection in the community and its major complication of PID. As an additional part of our hospital surveillance programme, we determined the threeyear moving average incidence of PID recorded in the $\mathrm{CMH}$ disease index (diagnosis at discharge) from 1968 to 1976 (Figure). The community surveillance programme was initiated in 1968. These

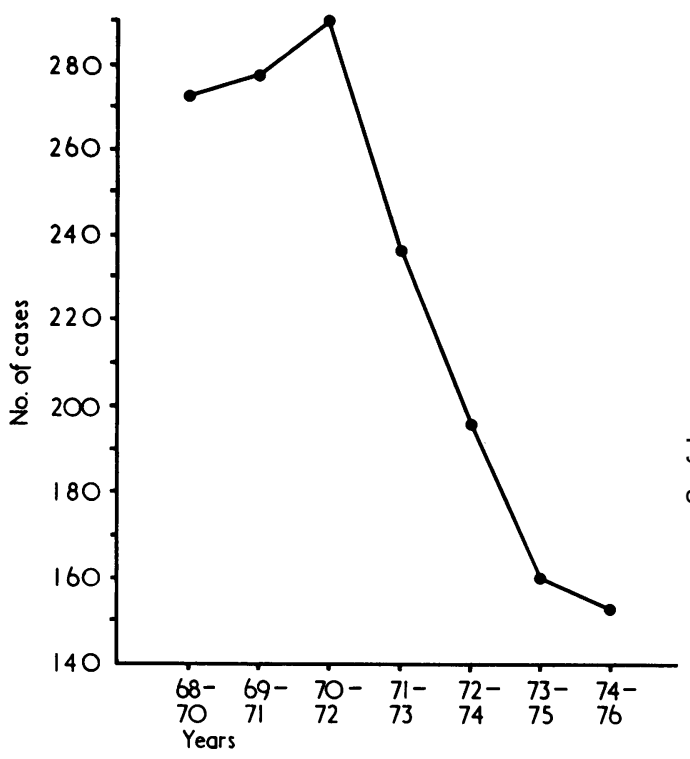

Figure Pelvic inflammatory disease for City of Memphis Hospital by three-year moving average (1968-76).

averages show an impressive decline in the number of cases of PID during this period. This becomes even more significant with the increased number of cultures taken in the latter years, and the decrease in the numbers and in the percentage of positive cases. Our data, therefore, do indicate that the control programme has had some impact on hospitalised cases of PID.

Our study is at variance with that of Stage et al. (1976) who, in a similar community and hospital, performed a retrospective study on patients admitted with a diagnosis of acute and chronic salpingitis, and found there was a $70 \%$ increase after two years of screening. This was despite an extensive community effort to identify and treat asymptomatic women with gonorrhoea and their 
male contacts, as was done in Memphis. However, Pedersen (1973) found a screening programme with private practitioners was successful in decreasing the incidence of PID.

Our data from Memphis indicate that the overall incidence of gonococcal infection in the study population remained unchanged between the first and second study periods. This indicates that the infection rate in women has stabilised; this is a departure from the recent continuous upward trend of reported cases of gonorrhoea in the United States of America (Center for Disease Control, 1976). More significant, in our opinion, is the impressive reduction we observed in hospitalised complications resulting from this disease. It is conceivable that a continuing surveillance programme for gonorrhoea in women, reaching a large segment of the population at risk, may have no impact upon the infection rate, which is primarily a behavioural problem, but it may nevertheless stop the infection from progressing to PID, by treating early infections. It is possible that we are witnessing this in our community. It would be a notable achievement in the control of gonorrhoea if we could reduce the incidence of PID to the same extent that the incidence and costs of institutional care for syphilis of the central nervous system have been reduced in the successful effort against that disease.

Further studies are in progress to find out if our continuing surveillance programme is resulting in fewer cases of gonorrhoea.

We wish to thank Joyce A. Wong for obtaining the disease index data.

\section{References}

Center for Disease Control (1976). VD Fact Sheet: Basic Statistics on the Venereal Disease Problem in the United States, thirty-third edition. Publication No. (CDC) 77-8195. Department of Health, Education, and Welfare: USA.

Pedersen, A. H. B. (1973). Screening program had definite impact in stemming infection. Canadian Medical Association Journal, 109. 1047-1048.

Rendtorff, R. C., Curran, J. W., Chandler, R. W., Glassco, S., and Wiser, L. (1976). The role of the urban community hospital in gonorrhoea surveillance. British Journal of Venereal Diseases, 52, 102-104.

Rendtorff, R. C., Curran, J. W., Chandler, R. W., Wiser, W. L., and Robinson, H. (1974). Economic consequences of gonorrhea in women: Experience from an urban hospital. Journal of the American Venereal Disease Association, 1, 40-47.

Stage, A. H., Brooks, G. G., Sanders, Z. G., and Waterfallen, J. W. (1976). A retrospective study on the effectiveness of a screening effort to reduce the complications of gonorrhea. Journal of the American Venereal Disease Association, 3, 61-64. 